\title{
From the Golden Ratio to the Golden Series and Their Social Application
}

\author{
Koumbakis Basilios ${ }^{1}$ \\ ${ }^{1}$ Mechanical Engineer, Teacher in Secondary Education from Thessaloniki, Greece \\ Correspondence: Koumbakis Basilios, Mechanical Engineer, Teacher in Secondary Education from Thessaloniki, \\ Greece. E-mail: koumbakis@gmail.com
}

Received: October 10, 2021

Accepted: November 4, 2021

Online Published: November 13, 2021

doi:10.20849/jed.v5i3.947

URL: https://doi.org/10.20849/jed.v5i3.947

The paper was presented at the 7th International Conference for Innovation in Education on 15-10-2021 in Larissa-Greece.

\begin{abstract}
This paper is about the logic of golden ratio. It is about the calculation of its value and the inverse value, examination of its uniqueness, the relation with Fibonacci sequence and its spiral and the logic of development of an organism. We expand the logic of golden ratio up until the sequence of Zeno from Elea that tends to infinity. We find the differentiate logic of golden ratio coming from ancient years and its unknown relation to the golden ratio. Also, we calculate the values $\varphi$ of series that follows the logic of golden ratio, reaching the golden (normal) series, as a result of its logic, with its modern applications. Finally, it is criticized the fact that we do not include golden ratio in our education and the consequences that this has, by compare it with the achievements of its era. The application of golden ratio's logic in social sciences results in possible examples of its use and their advantages.
\end{abstract}

Keywords: golden ratio, Fibonacci sequence, natural science, physics, social science

\section{Introduction}

Mathematical tools are been used in different fields of study and sciences in order to solve different problems. In some science applications the use of those tools has been admirably effective. A great example is the application of the golden ratio in Architecture. In Ancient Hellas the golden ratio was considered to be the expression of the inner harmony of nature and it was used as a mean of analogy in constructions, as would be the logic sense of nature. It is known from very old times, from geometric times, from the Egyptian pyramids and with its use were created amazingly beautiful structures that we admire even today. The purpose of this work is to utilize the existing knowledge in relation to the golden ratio, to interconnect independent parts of it, to broaden it and apply it to new fields such as the social field. By studying the golden ratio we find that it is used in one-dimensional quantities, like length and it is not used in two-dimensional or three-dimensional quantities, like surfaces or masses. Therefore, the study of the logic of the golden ratio has not been exhausted, so it is possible to extend it and apply it to fields other than the established ones.

\section{Golden Ratio}

The golden ratio is the intersection of a straight line length $\boldsymbol{\gamma}$ into two parts $\boldsymbol{\alpha}$ and $\boldsymbol{\beta}$

so that it is valid that $\boldsymbol{\alpha}+\boldsymbol{\beta}=\gamma$ and $\frac{\alpha}{\beta}=\frac{\beta}{\gamma}=\boldsymbol{\beta}$
where $\quad \gamma=$ the length of a straight line
$\boldsymbol{\alpha}=$ the length of first part
$\boldsymbol{\beta}=$ the length of second part
$\boldsymbol{\varphi}=$ the coefficient of ratio $\varphi=\alpha / \boldsymbol{\beta}$


(letter $\varphi$ is the initial letter of the Hellenic name "Phidias", as an honor of the architect of the Parthenon).

The golden ratio is calculated by solving the equations $\boldsymbol{\alpha}+\boldsymbol{\beta}=\boldsymbol{\gamma}$ and $\frac{\alpha}{\beta}=\frac{\beta}{\gamma}=\varphi$ setting the length of the linear $\operatorname{part} \gamma=1$, then $\frac{\beta}{\gamma}=\frac{\beta}{1}=\varphi$ so the $\boldsymbol{\beta}=\boldsymbol{\varphi}$.

We also have $\frac{\alpha}{\beta}=\varphi$ but $\beta=\varphi$ then $\frac{\alpha}{\varphi}=\varphi \quad=\quad \alpha=\varphi^{2}$

If we replace the $\boldsymbol{\alpha}$ and $\boldsymbol{\beta}$ in the equation $\boldsymbol{\alpha}+\boldsymbol{\beta}=\boldsymbol{\gamma}$ and we write the equivalent of $\varphi^{2}+\varphi=1$

or in normal form the quadratic equation $\varphi^{2}+\varphi-1=0$

The discriminant of equation it is $\Delta=\mathrm{b}^{2}-4 \mathrm{a} \mathrm{c}=1^{2}-4.1 \cdot(-1)=1+4=5 \quad$ and because $\Delta>0$ is positive that means that the equation has two roots $\varphi_{1} \& \varphi_{2}=\frac{-\mathrm{b} \pm^{2} \sqrt{\left(b^{2}-4 a c\right)}}{2 a}$

$$
\begin{gathered}
\varphi_{1}=\frac{-\mathrm{b}+{ }^{2} \sqrt{\left(b^{2}-4 \mathrm{ac}\right)}}{2 \mathrm{a}}=\frac{-1+{ }^{2} \sqrt{\left(1^{2}-4.1 \cdot(-1)\right)}}{2.1}=\frac{-1+\sqrt{2} \sqrt{5}}{2}=\mathbf{0 , 6 1 8} \\
\varphi_{2}=\frac{-\mathrm{b}-{ }^{2} \sqrt{\left(b^{2}-4 \mathrm{ac}\right)}}{2 \mathrm{a}}=\frac{-1-{ }^{2} \sqrt{\left(1^{2}-4.1 \cdot(-1)\right)}}{2.1}=\frac{-1-{ }^{2} \sqrt{5}}{2}=-\mathbf{1 , 6 1 8}
\end{gathered}
$$

The root $\varphi_{2}$ is negative while the lengths are positive, this means that the root $\boldsymbol{\varphi}_{2}$ is not real solution of equation $\boldsymbol{\varphi}^{2}+\boldsymbol{\varphi}-\mathbf{1}=\mathbf{0}$ and remains as a solution of the equation the $\boldsymbol{\varphi}=\boldsymbol{\varphi}_{1}=0,618$

then $\boldsymbol{\beta}=\boldsymbol{\varphi}$ the length of part $\boldsymbol{\beta}$ is $\boldsymbol{\beta}=0,618$

and $\boldsymbol{\alpha}=\boldsymbol{\varphi}^{2}$ the length of part $\boldsymbol{\alpha}$ is $\boldsymbol{\alpha}=0,618^{2}=0,382$

For verification $\alpha+\beta=\gamma=\mathbf{0 , 3 8 2}+\mathbf{0 , 6 1 8}=1$

The initial relationship of sections $\boldsymbol{\alpha}$ and $\boldsymbol{\beta}$ can be written upside down $\frac{\gamma}{\beta}=\frac{\beta}{\alpha}=\varphi^{\prime}=\frac{1}{\beta}=\frac{\beta}{\alpha}$

Then $\beta=\mathbf{1} / \varphi^{\prime}$ and $\alpha=\beta / \varphi^{\prime}=\mathbf{1} / \varphi^{, 2}$ converting the equation $\alpha+\beta=\gamma$ in $\varphi^{, 2}-\varphi^{\prime}-1=0$

and the root is:

$$
\begin{gathered}
\varphi_{1}^{\prime}=\frac{-\mathrm{b}+{ }^{2} \sqrt{\left(b^{2}-4 \mathrm{ac}\right)}}{2 \mathrm{a}}=\frac{1+{ }^{2} \sqrt{\left(-1^{2}-4.1 .(-1)\right)}}{2.1}=\frac{1+{ }^{2} \sqrt{5}}{2}=\mathbf{1 , 6 1 8} \\
\varphi_{2}^{\prime}=\frac{-\mathrm{b}+{ }^{2} \sqrt{\left(b^{2}-4 \mathrm{ac}\right)}}{2 \mathrm{a}}=\frac{1+{ }^{2} \sqrt{\left(-1^{2}-4.1 .(-1)\right)}}{2.1}=\frac{1-{ }^{2} \sqrt{5}}{2}=-\mathbf{0 , 6 1 8}
\end{gathered}
$$

The solution $\varphi^{\prime}{ }_{2}$ is negative and so it remains as a real solution $\varphi^{\prime}=\varphi^{\prime}{ }_{1}=1,618$

Consequently $\boldsymbol{\beta}=1 / \varphi^{\prime}=1 / 1,618=0,618$ and $\boldsymbol{\alpha}=\beta / \varphi^{\prime}=0,618 / 1,618=0,382$

The well-known since the time of Pythagoras, separation a part or a totality in parts that measure $\boldsymbol{\alpha}=\mathbf{0 , 3 8 2}$ and $\boldsymbol{\beta}=\mathbf{0 , 6 1 8}$ of totality $\gamma=1$ are the parts of the golden ratio.

The results of the two solutions $\varphi=0,618$ and $\varphi^{\prime}=1,618$ are interesting while related to each other $\varphi^{\prime}-\varphi=1$ $(1,618-0,618=1)$ and $\varphi^{\prime} * \varphi=1 \quad(1,618 * 0,618=1) \quad$ which means $\varphi^{\prime}-\varphi=\varphi^{\prime} * \varphi=1$

Also valid $\varphi^{\prime}-\beta=1$ and $\varphi^{\prime} * \beta=1$ which means $\varphi^{\prime}-\beta=\varphi^{\prime} * \beta=1$ or

$$
1,618-0618=1,618 * 0,618=1
$$

The unusual result for the upper equations indicates the uniqueness of the number $\varphi$.

\subsection{The Solution of the Golden Ratio in Antiquity}

The Figure 1 shows the geometrical solution of the intersection of a rectilinear part A-B into two sections, which have the golden ratio, as it was known in antiquity, 


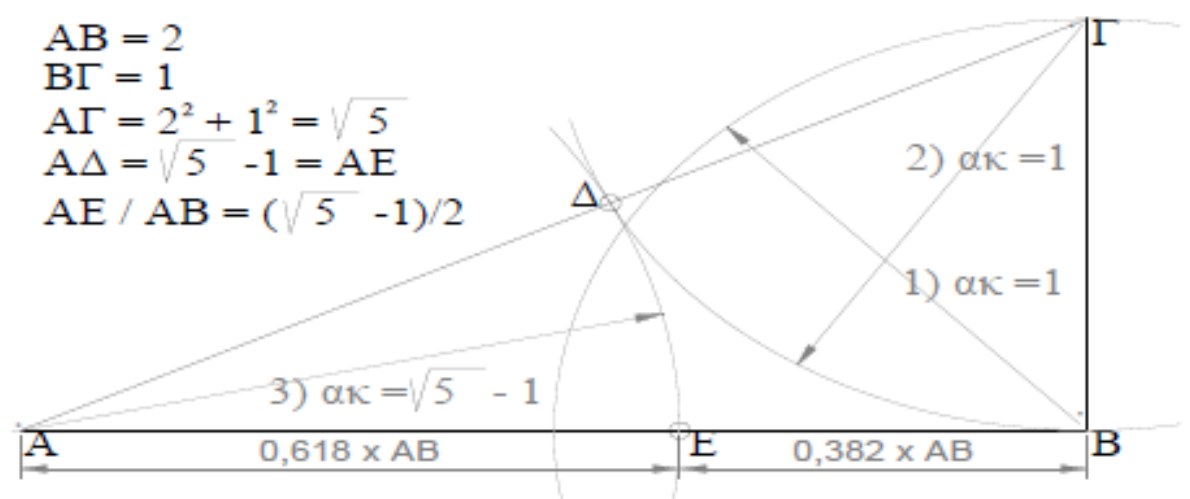

Figure 1. Geometric solution of the intersection of a line part A-B in two parts

\subsection{The Naturalness of the Golden Ratio}

The mathematical ratio $\varphi=0,618$ and $\varphi^{\prime}=1,618$ can been seen in nature, for example the ratio between the branches in plants, the bones in animals, the ratio in the development of spirals in shells and snails, the ratio of lengths in the human body also hold the analogy of $\boldsymbol{\varphi}$. The ratio $\boldsymbol{\varphi}$ was used in constructions in Ancient Hellas, like Parthenon of Athens which is considered one of the most beautiful architectural monuments, in the Aphrodite of Milos and in many more creations in Ancient Hellas and in Mediterranean during the Hellenistic period, with miraculous results of expression of their perfection, of their beauty, of harmony with nature as if it is an extension of its own logic.

Nature itself seems to choose this ratio $\varphi$ by equilibrate the countervailing forces of different parameters into harmony which in our eyes seems like beauty. The beauty is a relative value. We consider what we are used to seeing as beautiful. Because the first thing we see when we come to life and see it for a long time is nature, it enters as a model in the recordings of our brain and so that it has its own logic, its own proportions we consider it beautiful. We could name the ratio $\varphi$ natural ratio and not golden ratio as it has more to do with nature than gold.

\section{Golden Ration and Fibonacci Sequence}

It is impressive the relation of golden ratio $\varphi$ with Fibonacci sequence, in which every next number of the sequence is the sum of the two preceding numbers $\boldsymbol{\alpha}_{\mathbf{n}}=\boldsymbol{\alpha}_{\mathbf{n}-\mathbf{1}}+\boldsymbol{\alpha}_{\mathbf{n}-2}$ such as

$0,1,1,2,3,5,8,13,21,34,55,89,144,233,377,610,987,1597,2584 \ldots . . . e t c$.

So we conclude easily that the quotient of (two) consecutive numbers of the Fibonacci sequence as it develops, tends towards $\varphi$ or $\varphi$ ' respectively, such as

$$
\begin{aligned}
& \frac{2}{3}=0,666 ; \frac{3}{5}=0,6 ; \frac{5}{8}=0,625 ; \frac{8}{13}=0,615 ; \frac{13}{21}=0,619 ; \frac{21}{34}=0,617 ; \frac{34}{55}=0,618 ; \frac{55}{89}=0,618 ; \frac{89}{144}=0,618 \\
& \text { and } \\
& \frac{3}{2}=1,50 ; \frac{5}{3}=1,66 ; \frac{8}{5}=1,60 ; \frac{13}{8}=1,625 ; \frac{21}{13}=1,615 ; \frac{34}{21}=1,619 ; \frac{55}{34}=1,617 ; \frac{89}{55}=1,618 ; \frac{144}{89}=1,618
\end{aligned}
$$

It is also impressive in music for example, that one octave of 13 steps consists of 8 notes with 5 semitones $(5,8,13$ parts of the sequence) and the tones are parts of the diatonic and chromatic scale. In that way, today we have the opportunity to create music by the rules of math and submitting to ratio $\boldsymbol{\varphi}$.

The spiral based on Fibonacci sequence is constructed from squares, the sides of which are numbers of the sequence, joined by arcs with radius equal to their sides. 


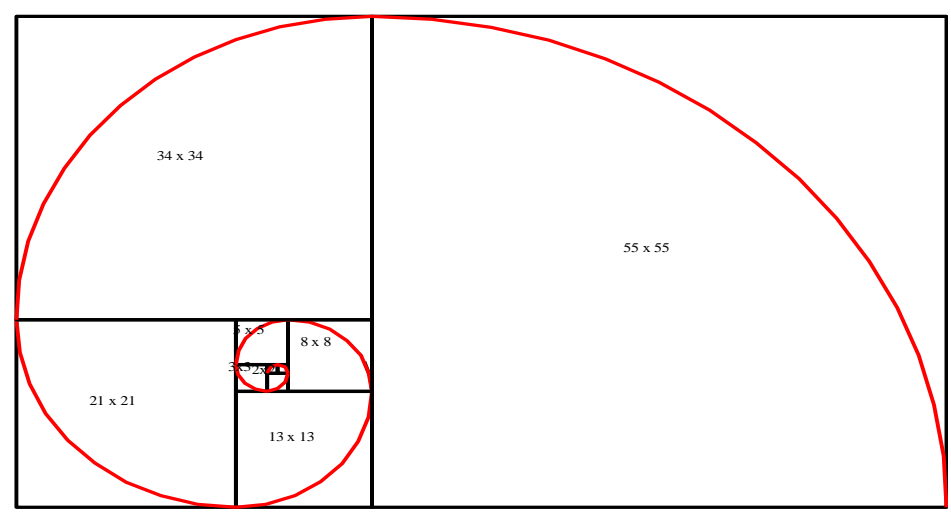

Figure 2. Fibonacci squares and their spiral

Also, the ratio of its consecutive squares follows the same by pointing towards the second part of the golden ratio the $\varphi^{2}=0,382$.

$$
\frac{3^{2}}{5^{2}}=0,36 ; \frac{5^{2}}{8^{2}}=0,39 ; \frac{8^{2}}{13^{2}}=0,378 ; \frac{13^{2}}{21^{2}}=0,383 ; \frac{21^{2}}{34^{2}}=0,381 ; \frac{34^{2}}{55^{2}}=0,382 ; \frac{55^{2}}{89^{2}}=0,382 ; \frac{89^{2}}{144^{2}}=0,382
$$

This spiral is been observed in natural processes like the evolution and growing of shells, plants, flowers, tornados, galaxies and so on. The Fibonacci sequence it is not only an interesting mathematical phenomenon, but it uncovers the way nature develops itself. By its definition, every next number of the sequence results from the sum of the two preceding ones, we can interpret that every stage of the development of an organism is based in the two preceding stages constituting their sum. This is not only about natural procedures - as it has already been observed - but the development in every natural and social field. The Fibonacci sequence can be interpreted as a continuation and application of Democritus' fundamental phrase "Nothing is born of nothing..." so to understand that everything is created from the pre-existing.

\section{Expanded Concept of Golden Ratio}

It is reasonable to ask the following questions:

The golden ratio is the only case of nature's "logic"?

Can this "logic" be extended to other applications?

What is the ratio $\varphi$ when a section is divided into more than two parts?

How can the totality of different parts be in inner balance, harmony?

\subsection{Enlargement in Many Sections}

We suppose that $\boldsymbol{\delta}$ is a straight part divided into three parts $\boldsymbol{\alpha}, \boldsymbol{\beta}$ and $\boldsymbol{\gamma}$

$\delta$

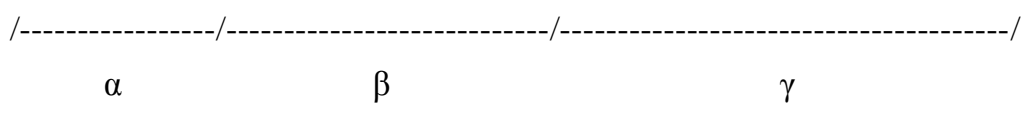

so that it is true that $\boldsymbol{\alpha}+\boldsymbol{\beta}+\boldsymbol{\gamma}=\boldsymbol{\delta}$ and $\frac{\alpha}{\beta}=\frac{\beta}{\gamma}=\frac{\gamma}{\delta}=\varphi$ where $\boldsymbol{\varphi}=$ coefficient the ratio

We set that the length of the linear part $\boldsymbol{\delta}=1$ unit of length

then $\gamma / \boldsymbol{\delta}=\gamma / \mathbf{1}=\boldsymbol{\varphi}$ which it comes from that the $\gamma=\boldsymbol{\varphi}$

and $\beta / \gamma=\varphi$ but after the $\gamma=\varphi$ then $\beta=\varphi^{2}$

in continuation $\alpha / \beta=\varphi$ but after the $\beta=\varphi^{2}$ then $\alpha=\varphi^{3}$

replaced the $\alpha+\beta+\gamma=\delta$ and generate the equivalent equation $\varphi^{3}+\varphi^{2}+\varphi^{1}=1$

or in normal form for the cubic equation $\varphi^{3}+\varphi^{2}+\varphi^{1}-1=0$

The cubic equation it should have three roots $\varphi_{1}, \varphi_{2}$, and $\varphi_{3}$

Continuing with the same reasoning, dividing a straight section $\varepsilon$ into four parts, 
We have $\boldsymbol{\alpha}+\boldsymbol{\beta}+\gamma+\boldsymbol{\delta}=\boldsymbol{\varepsilon}$ and $\frac{\alpha}{\beta}=\frac{\beta}{\gamma}=\frac{\gamma}{\delta}=\frac{\gamma}{\delta}=\varphi$. In this way we make a $4^{\text {th }}$ degree equation $\varphi^{4}+\varphi^{3}+\varphi^{2}+\varphi^{1}-1=0$ or an equation of such degree as the parts of the section.

Such equations of high degree cannot be solved using conventional methodology and for that reason we take non-conventional solutions for the quantification of $\varphi$ in every equation.

We observe that the equations $1=\varphi^{1}+\varphi^{2}, \quad 1=\varphi^{1}+\varphi^{2}+\varphi^{3}, 1=\varphi^{1}+\varphi^{2}+\varphi^{3}+\varphi^{4}$ consist of members with the form of members-a geometric progression $\varphi^{0}, \varphi^{1}, \varphi^{2}, \varphi^{3}, \varphi^{4}, \varphi^{5}, \ldots \ldots . \varphi^{\mathrm{n}}$ with a typical terms $\varphi_{\mathrm{n}}=\varphi^{*} \varphi^{\mathrm{n}-1}$.

Where $\varphi=$ ratio of geometric progression

$\mathbf{n}=$ the number of members of the geometric progression

Extending that logic, we find a series of infinite section parts of a straight-line segment or a totality

$$
\boldsymbol{\alpha}+\boldsymbol{\beta}+\boldsymbol{\gamma}+\boldsymbol{\delta}+\boldsymbol{\varepsilon}+\zeta+\boldsymbol{\eta}+\boldsymbol{\theta}+\ldots \text { toward infinity..... }=\mathbf{1}
$$

In which is valid $\frac{\alpha}{\beta}=\frac{\beta}{\gamma}=\frac{\gamma}{\delta}=\frac{\delta}{\varepsilon}=\frac{\varepsilon}{\zeta}=\frac{\zeta}{\eta}=\frac{\eta}{\theta}=\cdots$ toward infinity $\cdots=\varphi$.

We can create equations for the cut of a straight-line part or a totality in as much parts as we want, from the two that define the golden ratio until to infinite sections. All these cases could be expressed as equations of a geometric progression's sum with $\mathbf{n}$ parts that are show in the following table.

Table 1. The equations with the golden ration logic

\begin{tabular}{llc}
\hline Sections & \multicolumn{1}{c}{ the corresponding equation } & $\varphi$ \\
\hline 1 & $1=\varphi^{1}$ & 1 \\
\hline 2 & $1=\varphi^{1}+\varphi^{2}$ & $;$ \\
\hline 3 & $1=\varphi^{1}+\varphi^{2}+\varphi^{3}$ & $;$ \\
\hline 4 & $1=\varphi^{1}+\varphi^{2}+\varphi^{3}+\varphi^{4}$ & $;$ \\
\hline 5 & $1=\varphi^{1}+\varphi^{2}+\varphi^{3}+\varphi^{4}+\varphi^{5}$ & $;$ \\
\hline 6 & $1=\varphi^{1}+\varphi^{2}+\varphi^{3}+\varphi^{4}+\varphi^{5}+\varphi^{6}$ & $;$ \\
\hline 7 & $1=\varphi^{1}+\varphi^{2}+\varphi^{3}+\varphi^{4}+\varphi^{5}+\varphi^{6}+\varphi^{7}$ & $;$ \\
\hline 8 & $1=\varphi^{1}+\varphi^{2}+\varphi^{3}+\varphi^{4}+\varphi^{5}+\varphi^{6}+\varphi^{7}+\varphi^{8}$ & $;$ \\
\hline 9 & $1=\varphi^{1}+\varphi^{2}+\varphi^{3}+\varphi^{4}+\varphi^{5}+\varphi^{6}+\varphi^{7}+\varphi^{8}+\varphi^{9}$ & $;$ \\
\hline 10 & $1=\varphi^{1}+\varphi^{2}+\varphi^{3}+\varphi^{4}+\varphi^{5}+\varphi^{6}+\varphi^{7}+\varphi^{8}+\varphi^{9}+\varphi^{10}$ & $;$ \\
\hline
\end{tabular}

The solution of these equations is done according to the property of geometric progression's sum with the number of members, as many as the segments in which the totality equals 1 .

$$
\sum_{n=1}^{n}=\frac{\varphi\left(1-\varphi^{n}\right)}{1-\varphi}=1 \text { when } \varphi<1
$$

where $\quad \mathrm{n} \quad$ - is the parts in which a straight line or a totality is divided

$\boldsymbol{\varphi}$ - is the characteristic ratio of the geometric progression

$\Sigma_{\mathrm{n}}$ - is the sum of parts in which is divided the totality that is always one

When a totality is divided into infinite parts, then it is true that:

$$
\sum_{n=1}^{\infty} \varphi^{n}=\frac{\varphi}{1-\varphi}=1 \text { and solving it follows that } \varphi=1-\varphi \Rightarrow 2 \varphi=1 \Rightarrow \varphi=1 / 2
$$


Between the division of a totality into two parts and into infinite parts, there are the cases in between them which are shown in the following table and diagram. The following values in Table 2 have been calculated by software in a computer. The ratio $\varphi$ in all series is $\varphi<1$ which means that the series are converging.

Table 2. Values of the members of geometric progression with the logic of the golden ratio

\begin{tabular}{cccccccccccc}
\hline $\mathrm{N}$ & $\varphi_{1}$ & $\varphi_{2}$ & $\varphi_{3}$ & $\varphi_{4}$ & $\varphi_{5}$ & $\varphi_{6}$ & $\varphi_{7}$ & $\varphi_{8}$ & $\varphi_{9}$ & $\varphi_{10}$ & $\Sigma$ \\
\hline 1 & 1,000 & & & & & & & & & & 1,00 \\
\hline 2 & 0,618 & 0,382 & & & & & & & & & 1,00 \\
\hline 3 & 0,544 & 0,296 & 0,161 & & & & & & & & 1,00 \\
\hline 4 & 0,519 & 0,269 & 0,140 & 0,072 & & & & & & & 1,00 \\
\hline 5 & 0,509 & 0,259 & 0,132 & 0,067 & 0,034 & & & & & & 1,00 \\
\hline 6 & 0,504 & 0,254 & 0,128 & 0,065 & 0,033 & 0,017 & & & & & 1,00 \\
\hline 7 & 0,502 & 0,252 & 0,127 & 0,064 & 0,032 & 0,016 & 0,008 & & & & 1,00 \\
\hline 8 & 0,501 & 0,251 & 0,126 & 0,063 & 0,032 & 0,016 & 0,008 & 0,004 & & & 1,00 \\
\hline 9 & 0,501 & 0,251 & 0,125 & 0,063 & 0,031 & 0,016 & 0,008 & 0,004 & 0,002 & & 1,00 \\
\hline 10 & 0,500 & 0,250 & 0,125 & 0,063 & 0,031 & 0,016 & 0,008 & 0,004 & 0,002 & 0,001 & 1,00 \\
\hline$\ldots . .$. & $\ldots$ & $\ldots$. & $\ldots$. & $\ldots .$. & $\ldots .$. & $\ldots .$. & $\ldots .$. & $\ldots \ldots$ & $\ldots \ldots$ & $\ldots \ldots$. & $\ldots$ \\
\hline $\mathrm{n}$ & 0,500 & 0,250 & 0,125 & 0,063 & 0,031 & 0,016 & 0,008 & 0,004 & 0,002 & 0,001 & 1,00 \\
\hline
\end{tabular}

where $\varphi_{1}, \varphi_{2}, \varphi_{3}$ are the values of all the members of the geometric progression and the $\mathbf{n}=$ the number of sections to linear part, or a totality

The 10-part series terms are the approximately same as the terms of the series of infinite sections. Therefore, intermediate values tend to remain almost the same until the order of infinite terms that are:

$$
\frac{1}{2^{1}}, \quad \frac{1}{2^{2}}, \quad \frac{1}{2^{3}}, \quad \frac{1}{2^{4}}, \quad \frac{1}{2^{5}}, \quad \frac{1}{2^{6}}, \quad \frac{1}{2^{7}}, \quad \frac{1}{2^{8}}, \quad \frac{1}{2^{9}}, \quad \frac{1}{2^{10}}, \ldots \ldots \text { etc }
$$

This can also be seen in the diagram in Figure 3 .

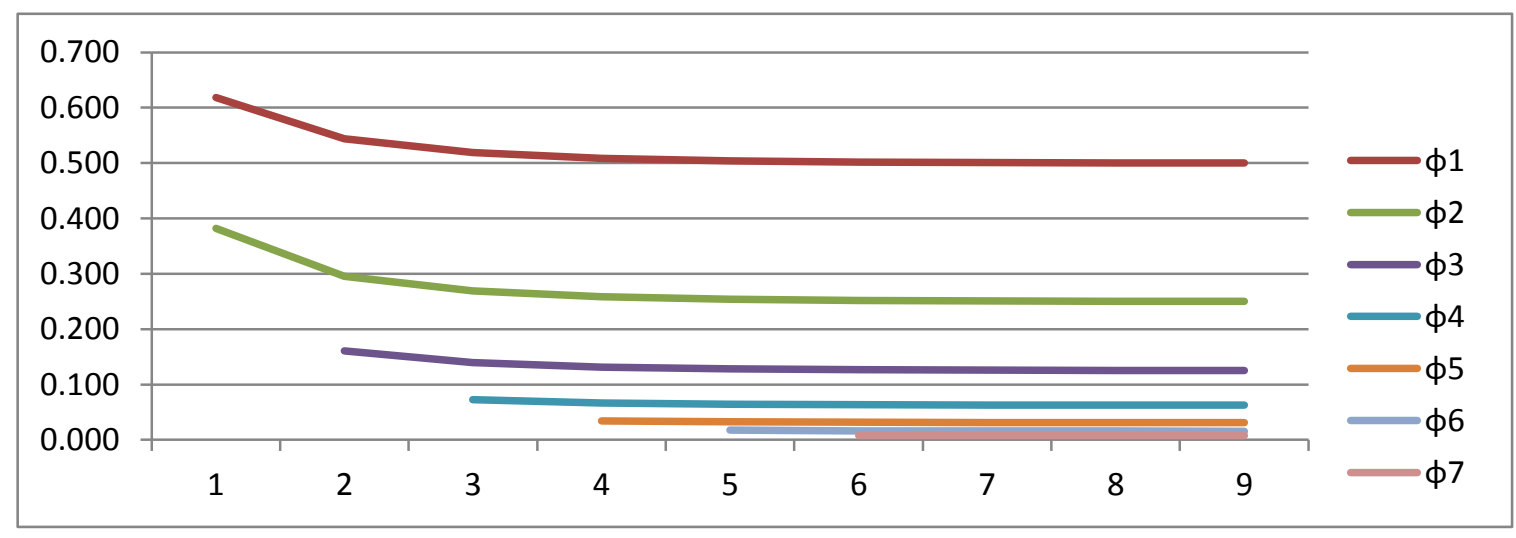

Figure 3. The terms $\varphi_{1}, \varphi_{2}, \varphi_{3}, \ldots \varphi n .$. of geometric progression in diagrammatic form

\subsection{Uniqueness of $\varphi$ and in Expanded Cases}

Table 2 is also showing the uniqueness of ratio $\varphi$ for these series e.g.:

A rectangle formed by two sections of a totality $\boldsymbol{\alpha}=0,382$ and $\boldsymbol{\beta}=0,618$ has surface $\alpha * \beta=0,618 \times 0,382=0,236$ which is exactly as the result of the subtraction $\alpha-\beta=0,618-0,382=0,236$.

A parallelepiped formed by 3 sections of a totality $\boldsymbol{\alpha}=0,544, \boldsymbol{\beta}=0,296$ and $\boldsymbol{\gamma}=0,161$ has a volume of 


$$
\begin{gathered}
\alpha * \beta * \gamma=0,544 \times 0,296 \times 0,161=0,026 \quad \text { which is exactly as the result of the subtraction } \\
\alpha * \beta-\beta * \gamma-\alpha * \gamma=0,544 \times 0,296-0,296 \times 0,161-0,544 \times 0,161=0,026
\end{gathered}
$$

A time-space entity formed by 4 sections of a totality $\alpha=0,519, \beta=0,269, \gamma=0,140, \delta=0,072$ has a value of

$$
\begin{gathered}
\alpha * \beta * \gamma * \delta=0,0014 \text { which is exactly as the result of the subtraction of their products } \\
(\alpha * \beta * \gamma)-(\beta * \gamma * \delta)-(\alpha * \beta * \delta)-(\alpha * \gamma * \delta)=0,019-0,002-0,010-0,0005=0,0014
\end{gathered}
$$

This characteristic is repeating in all series because it originates from property of the basic equation

$$
1=\varphi^{1}+\varphi^{2}+\varphi^{3}+\ldots+\varphi^{n}
$$

Golden (normal) series could be applied in all fields of mechanics like architecture, mechanical engineering, electrical engineering and also other fields like economics and sociology, in a way that expresses an internal harmony between the parts of a totality.

\section{The Golden (Normal) Series}

The last equation in Table 2 is the case of a straight part or a totality divided in infinite parts, following the logic of the same relation of the successive terms of the series as the golden ratio. It is known, by Zeno of Elea (488 - 425 B.C.), that the sum of series of infinite terms in a descending geometric progression with ratio $\varphi=1 / 2$ is equals with one (1).

$$
\Sigma=\left(\frac{1}{2}\right)^{1}+\left(\frac{1}{2}\right)^{2}+\left(\frac{1}{2}\right)^{3}+\left(\frac{1}{2}\right)^{4}+\left(\frac{1}{2}\right)^{5}+\left(\frac{1}{2}\right)^{6}+\left(\frac{1}{2}\right)^{7}+\left(\frac{1}{2}\right)^{8}+\left(\frac{1}{2}\right)^{9}+\cdots \ldots \ldots=1
$$

The sum of geometric progression of infinite terms is $\sum_{n=1}^{\infty} \varphi^{n}=\frac{\varphi}{1-\varphi}=1$ when $\varphi<1$

Where $\varphi=1-\varphi=>\varphi+\varphi=1 \Rightarrow 2 \varphi=1 \Rightarrow \varphi=1 / 2$ and the terms of this row is:

(0,500), (0,250), (0,125), (0,063), (0,032), (0,016), (0,008), (0,004), (0,002), (0,001), ....

Golden ratio and Zeno's sequence is the beginning and end of a natural logic. In other word the golden ratio is a section of segment in two parts while the Zeno's sequence is the section of a segment in infinite parts with the same logic.

From Zeno's sequence results another popular series which is widely used today. For an easier use of this, we take the derivatives of 10,100, 1000 etc of its terms and so we have the following series

$\begin{array}{llllllllll}1 & 1,25 & 1,6 & 2 & 2,5 & 3,2 & 4 & 5 & 6,3 & 8 \\ 10 & 12,5 & 16 & 20 & 25 & 32 & 40 & 50 & 63 & 80 \\ 100 & 125 & 160 & 200 & 250 & 320 & 400 & 500 & 630 & 800 \\ 1000 & 1250 & 1600 & 2000 & 2500 & 3200 & 4000 & 5000 & 6300 & 8000\end{array}$

and so on.

This series is known between engineers but perhaps it is not well-known that this is an extension of the golden ratio logic which use today is based on the regular series

$$
1,1.25,1.6,2,2.5, \quad 3.2, \quad 4,5,6.3,8, \quad 10,12.5,16 \ldots \text { etc }
$$

This series can be named "golden series" but because it has more to do with regularity, it would be better to name it "normal series". Golden ratio and normal series originate from the same logic.

The golden (natural) ratio and golden (normal) series come from the same logic, the division of a unity in parts with a specific logic, division of a part (totality) in two parts, such as golden ratio or in infinite parts, such as golden (normal) series.

\subsection{Examples of Application of the Golden (Normal) Series}

Various applications of this series are presented below.

Table 3. Standardization of tube diameters

\begin{tabular}{lllllllllllll}
\hline 10 & 15 & 20 & 25 & 32 & 40 & 50 & 63 & 80 & 100 & 125 & 160 & $\mathrm{~mm}$ \\
\hline $3 / 8^{\prime \prime}$ & $1 / 2 "$ & $3 / 4 "$ & $1 "$ & $11 / 4 "$ & $11 / 2^{\prime \prime}$ & $2 "$ & $21 / 2^{\prime \prime}$ & $3 "$ & $4 "$ & $5 "$ & $6 "$ & inch \\
\hline
\end{tabular}


Table 4. Standardization of nominal equipment pressures

\begin{tabular}{lllllllllllll}
\hline 6 & 10 & 12 & 16 & 20 & 25 & 32 & 40 & 50 & 63 & 80 & 100 & bar \\
\hline
\end{tabular}

Table 5. Standardization of cross-sections of electrical cables

\begin{tabular}{llllllllll}
\hline 1 & 1,5 & 2,0 & 2,5 & 4,0 & 6,0 & 8,0 & 10,0 & 16,0 & $\mathrm{~mm}^{2}$ \\
\hline
\end{tabular}

Table 6. Standardization of electrical fuse

\begin{tabular}{lllllllllllll}
\hline 10 & 16 & 20 & 25 & 32 & 40 & 50 & 63 & 80 & 100 & 125 & 160 & A \\
\hline
\end{tabular}

Table 7. Standardization of crane and vehicle loads

\begin{tabular}{lllllllllllll}
\hline 6,3 & 8 & 10 & 16 & 20 & 25 & 32 & 40 & 50 & 63 & 80 & 100 & ton \\
\hline
\end{tabular}

Table 8. Standardization of revolutions in machine tools

\begin{tabular}{lllllllllllll}
\hline 100 & 160 & 200 & 250 & 320 & 400 & 500 & 630 & 800 & 1000 & 1250 & 1600 & min- 1 \\
\hline
\end{tabular}

The properties of the regular series that make it preferable in electromechanical applications and enable many other applications are that:

- $\quad$ each member maintains the same relationship with its previous and next (by definition)

- $\quad$ in each member there is a next member of the series with 2 times its value

- $\quad$ in each member there is a next member of the series with 10 times its value

- in each member there is a next member of the row with the square of its value

- the difference between the successive values of the normal range is up to $12 \%$.

\section{Conclusions and Observations Golden Ratio Logic}

It is observed that the ratio $\varphi$ comes from nature and it is already used in natural sciences, biology, architecture, electrical engineering and mechanical engineering etc. The $\varphi^{\prime}=1,618$ or $\varphi=0,618$ are natural constants like the $\boldsymbol{\pi}=3,14159$ which was identified by Archimedes and the e $=2,71828$ which was determined by Bernoulli. The constant $\varphi$ though is not taught in school math as a constant that nature chooses in its development, balancing the opposite forces, as taught the other two physical constants $\boldsymbol{\pi}$ and $\mathbf{e}$.

Introducing the teaching of the golden ratio in the educational program in the classic and in its expanded sense will give future employees and future executives a tool to search for the best solution in their works. The golden ratio and the normal series, while being used in applications of natural sciences with good results, are not used in the field of social sciences and their applications where quantitative problem solving is generally not used. Efforts to solve social problems that arise (because for efforts are talked about and not solutions) are not made with mathematical tools but with legal and political tools, which often prove to be insufficient or inappropriate. Numerous legal texts, laws, decrees, regulations, circulars are required, proving the inadequacy of the applied problem-solving tools. This explains the backwardness of the social sciences in relation to the natural sciences and their applications.

\section{Comparative Example of Application of Natural and Social Sciences}

Today the travel of people and their constructions to space is just a routine, which was impossible to imagine 200 years ago, even someone with bold thinking, but for the 2500-year-old concept of democracy in Athenian society today efforts are still being made to implement it and this with shortcomings where its implementation has been partially achieved.

In problems that arise in the social field and in the conflicts that try to be solved through the social sciences, the logic of the golden ratio or the normal of series of things is not sought, but the maximum and the minimum as a solution to the problem. This concept of solving social problems without mathematical tools comes from the notion of maximalism and not from applications of scientific justification like golden ratio. It is attempted, that it is done in the social field to be at its maximum, e.g. or movement to be the largest, most massive, faster, the buildings 
higher, the constructions greater, the benefits of a process to be the maximum, the losses to be minimal and the profits the maximum, reaching up to be über alles.

From the results of application of the logic of maximalism it seems that they are achieved with the maximum positive results and the maximum problems that accompany them. Without overlooking the achievements of modern society, when we compare them with the achievements of antiquity and considering respectively the technological level, observed the lag in today's works.

The works of antiquity (material and non-material) from classical and Hellenistic period, despite the passage of thousands of years and many barbarian behaviors on them, remain works to admired from the whole world. This seems to be due not so much to the external result of the perishable works of the ancient works but to their interior, what their indestructible logic contains.

In this context we examine the ancient Hellenic phrase that traveled from ancient times up until today “ov̉к $\dot{\varepsilon} v \boldsymbol{\tau} \tilde{\varphi}$

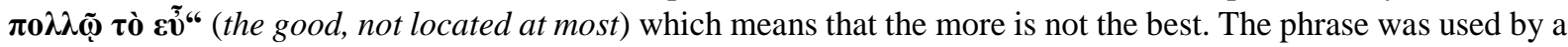
musician teacher called Kafisias, to his student who was playing his flute with extra volume but without achieving the desirable result in the end. Golden ratio is an applicable form of this phrase, without excluding other expressions and relation that manage in a different way inner harmony between different parameters of a totality, like the half of a value etc. The maximalistic logic of today is contrary to that ancient phrase and the golden ratio applications which is the aim of this paper.

The inner harmony of nature and then of the constructions following its logic is not met in social matters where the rivalry between standard, solid points of view does not leave room for solutions based on golden ratio, even if there would be an intention for this. Trying to convince an opponent of the validity of a point of view, is not the search for the truth, but proselytizing towards a point of view. It is far from the ancient phrase of Socrates " $\boldsymbol{\varepsilon} \boldsymbol{v} \boldsymbol{o} \boldsymbol{i} \boldsymbol{\delta} \boldsymbol{\alpha} \boldsymbol{\boldsymbol { o }} \boldsymbol{\boldsymbol { \sigma }} \boldsymbol{\boldsymbol { \imath }} \boldsymbol{\imath}$

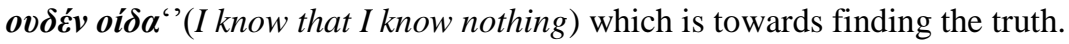

There is no education that can include these ideas and we are not teaching about the search of inner harmony in physics and social sciences, as well as we do not teach about golden ratio. We have it in the realm of mysticism and not in its mathematical explanation, integrated into the school curriculum with its applications. Likewise, we do not teach the dialectic method of Zeno as a method of solving problems, even if it is approximate solution, in the social sciences field. The relations $\boldsymbol{\varphi}$ in table 2 could possibly give solutions to social problems by analyze the inner structure, contrary to today's approach which is based in decrees and ineffective laws.

\subsection{Examples}

Example 1: Employees in primary sector, secondary and tertiary sector of economy.

If there are employees in primary sector $\geq$ employees in secondary sector $\geq$ employees in tertiary sector of economy, then there will be a lack of employees of higher qualification and technological knowledge which means that this sector will be less advanced resulting in reduced economic development of a country and creation conditions for job losses and people migrating.

If employees of primary, secondary and tertiary sector are distributing while maintain a ratio like the natural ratio between 3 parts of a totality, following the hierarchy of the enterprises of the first, second and third sectors of the economy, then based on the above mentioned there will be an equilibrium economy in county's, maintaining jobs by reducing immigration trends.

Example 2: Students in primary, secondary, tertiary degrees and master degrees

If students in primary school $=$ students in secondary (high school) $=$ students in tertiary degree (universities) $=$ students in master degrees, then in business market there will be a lack of lower-level work force and as a consequence higher cost of employees, higher cost of products, reduced competitiveness of businesses and of national economy with creation conditions of job losses and people migrating.

If the students between the 4 levels of studies had a ratio like the natural ratio of 4 parts of a totality, by following the organizational scale of production, then the competitiveness of businesses will increase so as the national economy and there will be less people for migrating.

Example 3: Income tax assessment scales.

If the income of an entrepreneur exceeds the lower limit of an income ladder, he will pay the same tax percentage as an entrepreneur who is at the upper limit of the same ladder. This creates a tendency to find interventions in the artificial transfer of income to the lower scale, thus tax evasion and reduction of government revenues. 
If the income scales are infinite (or enough to be considered infinite) as is actually the incomes of workers in a state, scaled by a ratio that the citizens would consider reasonable, then there will be no need to create bypass mechanisms of legal and tax provisions, maintaining in the real dimension the state revenues from taxes.

Teaching the logic of the golden ratio in the mathematics curriculum in secondary and tertiary education will give tomorrow's employees and executives, problem solving tools in the direction of finding the golden ratio of the opposing forces of everyday problems.

\section{References}

Archimedes from Wikipedia. Retrieved from https://el.wikipedia.org/wiki/П (mathematic constant

Bernoulli from Wikipedia. Retrieved from https://el.wikipedia.org/wiki/E_mathematicconstant

ELOT EN 10255 from Sidenor. Retrieved from https://sidenor.gr/proionta

Fibonacci from Wikipedia. Retrieved from https://en.wikipedia.org/wiki/Fibonacci_number

Isaac Cotes - Composing music with the Golden Ratio and the Fibonacci sequence. Retrieved from https://www.subaqueousmusic.com/geometry-in-music-composition-with-ableton-live/

Kaphisias from Sarantakos. Retrieved from https://sarantakos.wordpress.com/2016/05/27/eupolu/

Livio, M. (2003). The Golden Ratio. Broadways books, New York.

Pasioli, L. (1956). De Divina Proportione. Milano.

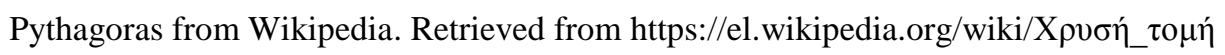

Stipancic, I., \& Matotek, J. (2010). The Golden Ratio. 14 International Conferences on Geometry and Graphic. Kyoto.

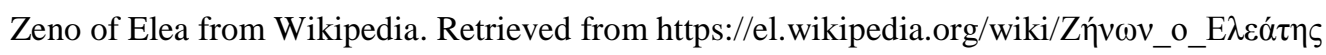

\section{Copyrights}

Copyright for this article is retained by the author(s), with first publication rights granted to the journal.

This is an open-access article distributed under the terms and conditions of the Creative Commons Attribution license (http://creativecommons.org/licenses/by/4.0/). 